\title{
VON WRIGHT SOBRE «AD IMPOSSIBILIA NEMO TENETUR»*
}

\author{
José Juan Moreso \\ Universitat Pompeu Fabra, Barcelona
}

Wenn er Pflicht ist, auch erreichbar sein muß. (KANT AA VIII, 1796: 418)

RESUMEN. En este artículo, el autor presenta las ideas centrales de vON WRIGHT acerca de la existencia de las normas, tal y como aparecen reconstruidas por ALCHOURRón y BULYGIN. A continuación se expone la reconstrucción del principio kantiano, debe implica puede, llevada a cabo por von WRIGHT. Se dan razones para aceptar la lectura de vON WRIGHT con arreglo a la cual el principio establece algunas de las condiciones de existencia de las normas. Contra ALCHOURRón y BULYGIN, se arguye que dicha idea comporta que, por razones conceptuales, los sistemas normativos no pueden contener normas contradictorias.

Palabras clave: Georg Henrik von Wirght, Carlos Alchourrón, Eugenio Bulygin, principio kantiano, existencia de normas, antinomias normativas.

\section{Von Wright on «Ad impossibilia nemo tenetur»}

ABSTRACT. In this paper, the author introduces the main ideas of VON WRIGHT on the existence of norms, as they are reconstructed by ALCHOURRÓN and BULYGIN. Then a reading of the so-called Kantian principle, ought entails can, is presented following VON WRIGHT. Some reasons are given in favour of the acceptance of this reading, in accordance which the principle put partial conditions for the existence of norms. Against ALCHOURRÓN and BULYGIN it is argued that such idea entails that, for conceptual reasons, normative systems cannot contain contradictory norms.

Keywords: Georg Henrik von WiRght, Carlos AlChOURRÓn, Eugenio BulYGin, Kantian principle, existence of norms, normative antinomies.

* Fecha de recepción: 22 de julio de 2016. Fecha de aceptación: 1 de septiembre de 2016.

Quiero agradecer los comentarios de mis amigos Pablo NAVARRO, Jorge RodrígueZ y Juan Ruiz MANERO a una primera versión de este trabajo, sin los cuales no habría recordado ni la relevancia de la réplica de von WRIGHT a los autores argentinos ni la importancia en Normative Systems de la noción de sistemas normativos puros, ni la necesidad de insistir en la relación entre la existencia y la racionalidad de los corpora normativos, entre otras cosas. Una versión de este trabajo fue presentado en una mesa redonda dedicada a conmemorar el centenario de von WRIGHT en el XII Congreso hispano-italiano-luso-francés de Teoría del Derecho en Lisboa, en junio de 2016, organizado por el Prof. DUARTE, al que quedo agradecido igual como a los demás participantes del congreso por sus sugerencias y comentarios, en especial a Eugenio BULYGIN que, aunque claramente en contra de lo que aquí defiendo, ha sido tan amable como siempre comentándolo de palabra y por escrito. La investigación se ha beneficiado de la ayuda financiera del Ministerio español de Economía y Competitividad y de la AGAUR de la Generalitat de Cataluña a los proyectos de investigación DER 2013-48066-C2-1-R y SGR 626. 2014-2, respectivamente. 


\section{INTRODUCCIÓN}

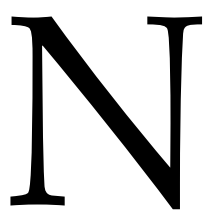

o cabe duda alguna de que Georg Henrik vON WRight (1916-2003) fue uno de los filósofos más relevantes de su tiempo, fue el sucesor de WITTGENSTEIN en la cátedra de Cambridge y uno de los tres albaceas de su legado intelectual. Sin embargo, tal vez porque regresó a Helsinki a mitad de los años cincuenta y permaneció desde entonces algo alejado de los focos filosóficos más relevantes de su tiempo (como el mismo Cambridge, Oxford o Harvard), su obra no es tan conocida como su agudeza y originalidad filosófica merecen. Pondré solo dos ejemplos: a) la vaguedad de nuestros conceptos vuelve a ser un tema central de análisis en la filosofía del lenguaje (y también en la metafísica y la epistemología actual), tal vez el libro fundamental de Timothy WiLLIAMSON (1994) tenga mucho que ver con ello. Sin embargo, en este debate VON WRIGHT suele estar ausente, a pesar de que a comienzos de los años ochenta publicó un trabajo (VON WRIGHT, 1984b) sobre las denominadas lógicas de la verdad que puede contemplarse como un enfoque semántico del problema que trata de hacer compatible la lógica clásica con la aceptación de proposiciones que carecen de valor de verdad. Contaré una anécdota al respecto: Pablo NAVARRO y yo llamamos la atención a Timothy ENDICOTT ${ }^{1}$ sobre este trabajo en Oxford en 1995, trabajo que él — como casi todo el mundo en Oxforddesconocía a pesar de haber sido publicado la década anterior por Basil Blackwell en Oxford. b) Muchos autores ahora reivindican la denominada teoría minimalista (también deflacionaria) de la verdad (véase el influyente HORWICH, 1990), pues bien se trata de una concepción anticipada por VON WRIGHT con argumentos similares a los de HORWICH también a comienzos de los ochenta del pasado siglo (VON WRIGHT, 1984a). Tampoco en este caso este precedente es casi nunca reconocido ${ }^{2}$.

Por fortuna, en el ámbito de la filosofía jurídica de los países latinos su obra ha tenido más lectores y un lugar central. Eso se debe fundamentalmente al hecho de que Carlos E. AlCHOURRÓN y Eugenio BULYGIN lo tomaron como inspiración de su teoría de los sistemas normativos (1971). Además AlCHOURRÓn y BuLYGIN escribieron en 1973 (AlCHOURRÓN-Bulygin, 2015: 90) un trabajo sobre la filosofía del derecho del pensador finlandés, un trabajo que para mí es un ejemplar afortunado de claridad, originalidad y agudeza filosófica. De hecho, cuando fui invitado a esta mesa redonda, creía que nada podía añadir al retrato fiel — que les sirve para presentar algunas ideas de su propia concepción - de los iusfilósofos argentinos. Sin embargo, hay un punto que considero relevante, y que ellos apuntan pero no desarrollan (ALCHOURRÓNBuLYGIN, 2015: 90), que es la relación entre el principio kantiano debe implica puede, o dicho como los juristas romanos ad impossibilia nemo tenetur, y la existencia de las normas.

Presentaré, en primer lugar, las ideas centrales de VON WRIGHT (1963: cap. 7) acerca de la existencia de las normas tal y como son reconstruidas por ALCHOURRÓN y

1 Como él reconoce amablemente en EndicotT (2000: 67 nota 28). Después Pablo NAVARRo, Cristina REDONDO y yo usamos las ideas de VON WRIGHT para tratar algunos problemas de teoría general del derecho, como las lagunas y las antinomias (MORESO-NAVARRO-REDONDO, 2001; 2002; 2003).

2 Ni en Horwich (1990), ni en, por ejemplo, la entrada de la Stanford Encyclopedia (STOLjaR-DamnjanoVIC, 2014) dedicada a la teoría deflacionista de la verdad, en los cuales VON WRIGHT está ausente. 
BuLYGIN (2015). En segundo lugar, me referiré al principio kantiano y a su significado con arreglo a las ideas de VON WRIGHT. En tercer lugar, presentaré algunas consecuencias que se siguen de estas ideas de VON WRIGHT acerca del principio y su imbricación con la noción de existencia de las normas. Después, me referiré a las consecuencias de la posición de VON WRIGHT para la cuestión de las antinomias en los sistemas normativos. Y, finalmente, concluiré.

\section{ALCHOURRÓN Y BULYGIN SOBRE VON WRIGHT}

Las ideas de VON WRIGHT sobre la existencia de las normas se hallan fundamentalmente en el cap. 7 de Norm and Action (1963). Aquí VON WRIGHT se refiere en especial a la noción de existencia de las prescripciones, de órdenes emitidas por ciertas autoridades y dirigidas a otras personas como destinatarios, los sujetos normativos ${ }^{3}$. En el siguiente pasaje (VON WRIGHT, 1983a: 68) el autor aclara aquello que las normas no son:

Es importante no confundir la norma misma con la formulación normativa, ni la existencia de una norma con la emisión u otra producción de una formulación normativa. No discutiré aquí la naturaleza lógico-semántica de la relación de la norma con su expresión en palabras. Solo deseo decir, al pasar, que no considero correcto denominar como norma ni la referencia, ni el significado, ni el sentido de la formulación normativa.

Esto hace algo enigmática la concepción de las prescripciones de VON WRIGHT ${ }^{4}$. Y lleva a ALCHOURRÓN-BULYGIN a decir (2015: 90-91) que no hay una única noción de prescripción. Podemos distinguir, sostienen, entre la comunicación, el acto de comunicar un contenido descriptivo, como, por ejemplo, cuando yo le digo a un amigo «el Real Madrid ganó la Champions League» que requiere la emisión de una oración por mi parte y la recepción por parte de mi destinatario, la aserción que yo pronuncio aún si no consigo comunicarla, digamos, porque mi amigo no me oye y la proposición, el contenido de una aserción posible, aún si nadie la ha emitido, la proposición que el Madrid ganó la Champions League en 2016. Según los autores, podemos distinguir, en las prescripciones, como cuando yo le digo a mi hija «Debes estar en casa antes de las nueve de la noche», entre la norma-comunicación (la orden dada y recibida por mi hija), la norma-prescripción (la orden promulgada con independencia de si ha sido recibida o no) y la norma-lekton (el contenido de un meramente posible acto de prescribir). Y ALCHOURRÓN y BULYGIN insisten (2015: 91-93) en que la noción de norma más útil en el derecho es la noción de norma-prescripción, es decir que una norma comienza a existir cuando es promulgada. Según los autores, cualquier acto serio de prescribir da lugar a la existencia de una norma. Su posición parece plausible y, por ende, su crítica a VON WRIGHT fundada. Sin embargo, creo que merece la pena indagar mejor en la noción de existencia de las normas de VON WRIGHT y tal vez la idea de un acto serio de prescribir sea la clave. VON WRIGHT (1963: 116-117) compara el acto

3 Prescindo aquí de las reflexiones del autor acerca de la importancia del principio debe implica puede para delimitar las condiciones de otro tipo de normas, como las reglas (que definen un juego, por ejemplo), las normas técnicas o las reglas ideales (vid. VON WRIGHT, 1963: 112-114).

4 En este sentido HERNÁNDEZ MARÍN (1986: 39-40). 
de prescribir con el acto de prometer y, convincentemente, nos recuerda que el hecho de que una persona emita un enunciado de promesa no lo convierte en promitente, no hace que exista una promesa. Si un niño, sigue VON WRIGHT, promete darnos mil libras mañana, no diremos que hay una promesa de la que somos promisarios. Las condiciones de existencia de las promesas son más complejas que la mera emisión de un enunciado de promesa (para ello VON WRIGHT, 1983b). Resultan en la generación de una normative relationship —una vinculación normativa - ${ }^{5}$ por un periodo de tiempo entre el promitente y el promisario. También, de modo similar, las condiciones de existencia de las normas son más complejas. Las normas existen en un sentido similar al que existen las promesas.

Creo que las ideas de VON WRIGHT pueden ser presentadas del siguiente modo:

Una norma -una prescripción- P existe si y solo si:

a) Una autoridad ha emitido una orden, que obliga o prohíbe, o una permisión.

b) Los destinatarios, los sujetos normativos, han recibido la orden o la permisión.

c) Los destinatarios, los sujetos normativos, de la orden o permisión pueden hacer aquello que está ordenado o permitido.

La primera cláusula de la definición hace la existencia de este tipo de normas dependiente de dos cosas, por una parte la existencia de las prescripciones es dependiente del lenguaje, hace falta que alguien emita determinados enunciados para que las prescripciones existan, por otra parte la existencia de las prescripciones depende de que su emisor sea de facto una autoridad, tenga la situación de superioridad que hace posible la generación del vínculo normativo. La segunda cláusula es necesaria también, si la existencia de las prescripciones consiste en la generación del vínculo normativo. La tercera cláusula supone lo siguiente (VON WRIGHT, 1963: 109): «que algo sea el contenido de una prescripción implica que el sujeto de la prescripción pueda hacerlo».

No insistiré en aspectos que tienen importancia referidos a las dos primeras cláusulas (que centran el análisis de VON WRIGHT), y me detendré en la tercera cláusula que, como vemos, es una formulación del principio kantiano. Para ello, detengámonos un poco en el significado de dicho principio.

\section{EL PRINCIPIO KANTIANO}

En la obra de Immanuel KANT hay varios pasajes que acreditan la razón de que el principio debe implica puede le sea atribuido. Junto con el que encabeza esta contribución hay otros varios, uno de los más famosos en la Crítica de la Razón Pura (A 548/ B576): «El acto al que se aplica el deber tiene que ser realmente posible bajo condiciones naturales» ${ }^{6}$. Hay un gran debate acerca del significado y el alcance de muchos

5 Esta es la afortunada expresión con la que Pedro GARCÍA FERRERO traduce «normative realtionship» en la versión española de Norm and Action (Norma y acción, Madrid: Tecnos, 1970), a la que, sin embargo, no sigo en otros puntos.

6 «Nun muß die Handlung allerdings unter Naturbedingungen möglich sein, wenn auf sie das Sollen gerichtet ist». (KANT, 1781/1787: A548/B576). 
de estos pasajes, STERN (2004) por ejemplo identifica hasta once textos relevantes de KANT al respecto.

Pero este no es un texto de hermenéutica kantiana, aquí solo deseo subrayar el rechazo de VON WRIGHT (1963: 109-110) de una posible lectura del principio de acuerdo con la cual el deber, la norma, implica lógicamente la capacidad de llevar a cabo aquello prescrito. Un modo de, como nos recuerda, rechazar la humeana separación entre el ser y el deber ser. Porque si que debo hacer A, implica lógicamente que puedo hacer A, entonces si no puedo hacer A (un hecho), no debo hacerlo (una norma). Pero vON WRIGHT (1963: 110) interpreta el principio de otro modo, como una relación entre una proposición normativa, que afirma la existencia de una norma y un hecho acerca de las capacidades humanas:

El principio que debe implica puede, como yo lo entiendo, no afirma una relación de implicación entre una norma y una proposición. La implicación se da entre proposiciones normativas (verdaderas o falsas), por una parte, y proposiciones acerca de las capacidades humanas, por otra. El antecedente (la premisa) establece que hay una norma con tal carácter y tal contenido. El consecuente (la conclusión) establece que la cosa mandada o permitida, que es el contenido de la norma, puede hacerse. Conforme a esta interpretación, el principio kantiano que debe implica puede no está en conflicto con la idea humeana de la independencia lógica entre el deber y el ser.

Ahora se comprende la importancia del principio kantiano para la noción de existencia de las normas del autor finlandés: lo que podemos hacer es una condición de la existencia de las prescripciones. Lo dice así (1963: 113): «El propósito del principio, como yo lo veo, es el de ayudar a moldear o configurar el concepto de norma».

A continuación me referiré a algunas de las consecuencias de esta idea.

\section{LA NOCIÓN DE CORPUS NORMATIVO}

Prescindamos ahora de muy relevantes cuestiones entrelazadas con la noción de VON WRIGHT de existencia de las prescripciones, como por ejemplo la referida a la dimensión temporal de las normas, el periodo de tiempo en el que una norma prescriptiva existe (ALCHOURRÓN-BULYGIN, 2015: 93). Y detengámonos en un aspecto, que afecta a la primera cláusula de nuestra definición, y que deseo enfatizar. Para vON WRIGHT, no solo existen las normas explícitamente promulgadas por una autoridad, sino también aquellas que estas implican (VON WRIGHT, 1963: 156): «Las prescripciones que son implicadas por un conjunto dado de normas las llamaré mandatos, prohibiciones y permisiones derivados». Y añade (1963: 157-8):

Los mandatos, prohibiciones y permisiones derivados de un corpus de prescripciones son, podríamos decir, tan «queridos» por la autoridad normativa como los mandatos, prohibiciones y permisiones originales de este corpus. Las normas derivadas están, necesariamente, en el corpus con las originales. Están allí, aunque no han sido expresamente promulgadas. Su promulgación se esconde en la promulgación de otras prescripciones.

Obviamente, esta idea es central en VON WRIGHT y articula la elegante reconstrucción de ALCHOURRÓN-BULYGIN (2015). Con arreglo a ella, a un corpus normativo pertenecen, y existen de este modo, no solo las normas promulgadas por actos de pres- 
cribir de las autoridades, las normas formuladas, sino también todas sus consecuencias lógicas, las normas derivadas ${ }^{7}$.

Para ello, necesitamos una noción de consecuencia lógica entre normas, de implicación normativa. Que se halla en VON WRIGHT (1963: 158- 167) y que ALCHOURRÓNBULYGIN reconstruyen de un elegante modo (2015: 101-111), con tres axiomas y una regla de inferencia. Ahora bien, ¿cuándo dos normas pertenecen al mismo corpus normativo?

Para VON WRIGHT (1963: 151), un corpus de normas es un conjunto de prescripciones emitidas por la misma autoridad. ¿Por qué VON WRIGHT establece esta restricción tan importante en la noción de corpus normativo? Porque considera que la consistencia de un conjunto normativo es una condición de la posibilidad de existencia de un corpus. Las normas incompatibles comportan la imposibilidad de su coexistencia en un corpus. Y, VON WRIGHT considera, esta exigencia de racionalidad solo puede establecerse como requisito de la voluntad de una autoridad individual. Es decir, nunca normas incompatibles pueden coexistir en el mismo corpus, aunque puedan existir en distintos corpora.

Para que esta noción de corpus sea viable, es preciso proporcionar una noción de consistencia entre normas que VON WRIGHT ofrece (1963:143-144) y que ALCHOURRÓN-BULYGIN (2015: 97) resumen de este modo:

¿Qué significa que dos o más normas sean mutuamente consistentes? VON WRIGHT da primero un criterio formal de consistencia o compatibilidad para las formulaciones normativas, esto es, para las expresiones deónticas O y P. La definición de consistencia formal está dada en tres pasos: para conjuntos de expresiones $\mathrm{O}$, para conjuntos de expresiones $\mathrm{P}$ y, finalmente, para conjuntos mixtos de expresiones $\mathrm{O}$ y $\mathrm{P}$. Un conjunto de expresiones $\mathrm{O}$ (mandatos) es consistente si, y solo si, «es lógicamente posible, bajo cualquier condición de aplicación dada, obedecer todos los mandatos (colectivamente) que se aplican bajo esa condición» (N\&A, 143). Un conjunto de expresiones P (permisiones) es siempre consistente. Porque las permisiones nunca se contradicen. Esto significa lo siguiente: si bien puede no ser posible hacer uso de todas las permisiones al mismo tiempo, siempre es posible hacer uso de cualquiera de ellos en un momento determinado. Un conjunto mixto (de mandatos y permisiones) es consistente «si y solo si, es lógicamente posible, bajo cualquier condición de aplicación dada, obedecer todos los mandatos colectivamente y hacer uso individualmente, de cada una de las permisiones que se aplican bajo esta condición» (N\&A, 144).

AlCHOURRÓn y BulYgin aceptan esta noción de consistencia de normas. Sin embargo, rechazan las consecuencias que VON WRIGHT obtiene de ella porque no comparten, principalmente, su noción de existencia de normas y tampoco su noción de corpus normativo. A los autores argentinos (AlCHOURRÓN-BULYGIN, 2015: 98-99) les parece arbitrario restringir la lógica de normas a normas emitidas por una y la misma autoridad, les parece problemática la noción de la «misma autoridad», por ejemplo ¿es

7 Esta idea es discutida perspicuamente, distinguiendo entre las normas explícitamente promulgadas que integran la base del sistema y las normas implícitamente promulgas que no lo integran, en NAVARRO-RODRÍGUEZ (2014: 214-240), para explorar las consecuencias que tiene para la dinámica de los sistemas jurídicos. Dado que aquí no me ocupo de ello, dicha idea no será analizada aquí. 
la misma autoridad el parlamento portugués de hoy que el de hace dos años?, les parece implausible decir que una autoridad es irracional por dictar normas inconsistentes en momentos temporales distintos y, finalmente, les parece que tiene como consecuencia más bien sorprendente que no pueden existir normas inconsistentes: en el mismo corpus porque se «aniquilan mutuamente», y en el caso de autoridades distintas, porque no hay aquí inconsistencia.

No voy a discutir aquí estas objeciones, aunque algunas podrían ser replicadas o matizadas. Voy a intentar mostrar que la noción de corpus normativo de VON WRIGHT tiene sentido, al menos, en el supuesto de las normas aplicables con las cuales el juez debe decidir un caso y motivar en el Derecho su decisión. Esta es precisamente la noción de sistema normativo de ALCHOURRÓN y BULYGIN en Normative Systems (1971). Como es sabido, se trata de seleccionar un universo del discurso (un conjunto de acciones humanas) y seleccionar las normas que lo regulan. En este conjunto, de acuerdo con los autores argentinos, puede haber contradicciones o antinomias ${ }^{8}$. Esta es la idea que deseo objetar, valiéndome de las ideas de VON WRIGHT.

Asumimos que los jueces motivan adecuadamente sus decisiones cuando muestran que de ese corpus de normas y de las circunstancias fácticas del caso se deriva una norma individual que resuelve dicho caso individual. Asumimos también que las normas son aptas para guiar el comportamiento de los jueces. Y añadimos la verdad lógica según la cual de aquello que es inconsistente se sigue cualquier cosa, ex falso quodlibet. Entonces, conjuntos inconsistentes de normas no pueden guiar el comportamiento de los jueces, porque tomen la decisión que tomen estaría de acuerdo con ese corpus de normas. Los corpora de normas inconsistentes no son aptos para discriminar entre soluciones correctas e incorrectas y, por lo tanto, no están sujetos a la racionalidad. En este sentido, no hay corpora normativos inconsistentes. Esta conclusión presupone desplazar la idea de corpus de normas, de las normas promulgadas por la misma autoridad a las normas aplicables, aptas para resolver un caso judicial.

En realidad, con posterioridad a Norm and Action, VON WRIGHT desarrolló esta idea de corpus racional de normas del siguiente modo (VON WRIGHT, 1983c: 140-141):

Si una norma, o un corpus, no es susceptible de ser satisfecha (consistente normativamente) el legislador o la autoridad normativa suprema (soberana) no puede racionalmente querer el estado ideal previsto en su actividad creadora de normas o legislación.

La condición de racionalidad que he establecido puede ser contemplada como una condición mínima necesaria. Pueden añadirse ulteriores condiciones, por ejemplo que la obtención de los varios estados que las normas hacen obligatorios o permitidos deba ser también físicamente posible, o humanamente posible.

Pues bien, lo que trataré de argüir a continuación es que los corpora de normas que contienen normas inconsistentes, necesariamente implican que hay estados de cosas que no pueden ser realizados por los destinatarios de las normas, porque implican todos los estados de cosas. Si se conciben estas condiciones como presupuestos de la existencia de los corpora normativos, entonces no hay corpora inconsistentes.

\footnotetext{
8 Aunque Normative Systems (1971: cap. VIII) contiene un capítulo dedicado al análisis del problema de las lagunas y la decisión judicial, no hay ninguno desafortunadamente dedicado a las antinomias y la decisión judicial.
} 
José Juan Moreso

\section{ANTINOMIAS Y CORPUS DE NORMAS}

En un influyente artículo Bernard WiLLIAms (1965), planteó el siguiente problema lógico para mostrar que una determinada forma de concebir las obligaciones morales no deja lugar para los dilemas. Supongamos que un corpus contiene una norma que obliga a hacer A, y otra norma que obliga a hacer B, y que A y B no son estados de cosas posibles (posibles lógica o físicamente, ahora es indiferente) conjuntamente. Entonces tenemos una situación como la siguiente:
1. $\mathrm{OA}$
Premisa
2. $\mathrm{OB}$
Premisa
3. $\neg \diamond(\mathrm{A} \wedge \mathrm{B})$
Premisa
4. $\mathrm{O} \alpha \rightarrow \diamond \alpha$
Principio kantiano
5. $\mathrm{OA} \wedge \mathrm{OB}$
De 1 y 2
6. $(\mathrm{OA} \wedge \mathrm{OB}) \rightarrow \mathrm{O}(\mathrm{A} \wedge \mathrm{B})$
Principio de aglomeración
7. $\mathrm{O}(\mathrm{A} \wedge \mathrm{B})$
De 5 y 6
8. $\mathrm{O}(\mathrm{A} \wedge \mathrm{B}) \rightarrow \diamond(\mathrm{A} \wedge \mathrm{B})$
Del principio kantiano en 4
9. $\neg \mathrm{O}(\mathrm{A} \wedge \mathrm{B})$
Tollendo ponens, en 7 y 8

Como vemos las tres plausibles premisas implican una contradicción (entre 7 y 9). ¿Cómo podemos bloquear la contradicción? En la literatura existe un rico y detallado debate con muchas vías de análisis alternativas (véase una presentación panorámica en McConell, 2014), que en mi opinión pueden ser reducidas a las tres siguientes:

i) Rechazar el principio de aglomeración.

ii) Rechazar el principio kantiano.

iii) Rechazar que en este corpus normativo puedan coexistir las normas contenidas en $1 . \mathrm{y} 2$.

Pero antes de analizarlas, es necesario recordar que VON WRIGHT considera que el principio kantiano contiene una implicación entre una proposición normativa, que afirma la existencia de ciertas normas con determinado contenido, y determinadas posibilidades fácticas. Es decir, que las dos primeras premisas de este argumento deben ser comprendidas, en la interpretación del filósofo finlandés, como «Existe un corpus normativo que obliga hacer A (o, en el caso de la segunda, $\mathrm{B}$ )». Y así todo el resto de líneas del argumento, con lo que la última debe ser leída como «No existe un corpus normativo que obligue a hacer A y B».

La primera vía, el principio de aglomeración, no merece mayores comentarios porque pocos están dispuestos a rechazarlo. Y menos que nadie VON WRIGHT (1951: 13) y ALCHOURRÓN y BULYGIN (2015: 102) para los cuales el principio de aglomeración es un axioma o un teorema indisputable de la lógica deóntica.

La segunda vía, el rechazo del principio kantiano, suele ir acompañado de la idea de que las obligaciones contenidas en las dos primeras premisas no son concluyentes, no son decisivas, sino solo pro tanto o prima facie. Esta línea da lugar a la rica literatura, en moral, y en lógica y en inteligencia artificial, relativa a los razonamientos no-monótonos y la derrotabilidad ${ }^{9}$. Tampoco será abordada aquí puesto que en los corpora

\footnotetext{
9 Puede verse, por todos, Celano (1994: 458-478) y AlChOURRÓN (1996).
} 
normativos disponibles por aquellos que han de tomar una decisión justificada, como es el caso de los jueces, precisamos de normas que establezcan razones decisivas, concluyentes, las únicas aptas para justificar en ellas las decisiones.

Y ello, nos deja, según creo con la tercera vía. El principio kantiano, entendido à la VON WRIGHT, comporta que no hay corpora normativos que impongan deberes no realizables. No hay, tampoco, corpora con normas incompatibles. De hecho, si hubiera corpora con normas incompatibles también violarían el principio kantiano, dado que si una norma obliga a hacer A, y otra obliga a hacer no-A y dado que es lógicamente imposible que A y no-A, entonces no puede haber corpora inconsistentes. Dicho de otro modo, si el principio kantiano es entendido como una condición de existencia de los corpora normativos, entonces no existen corpora normativos inconsistentes.

Esta me parece una consecuencia más que aceptable si los corpora son comprendidos como sistemas de normas dispuestos a ser aplicados por los jueces. Los jueces deben, entre dos prescripciones incompatibles promulgadas por las autoridades, seleccionar solo una de ellas para ser integrada en el corpus con el que tomará la decisión. Solo así puede aspirar a que su decisión sea racionalmente justificable de acuerdo con un corpus de normas. En este sentido, podemos restaurar la vieja idea ilustrada del legislador racional, un legislador que no genera antinomias. Y, también en este sentido, podemos aprovecharnos de una de las tantas ideas fecundas de VON WRIGHT, aunque sea al precio de distanciarnos un poco de AlCHOURRón y BulYGIN.

\section{RACIONALIDAD Y EXISTENCIA DE NORMAS}

De acuerdo con VON WRIGHT, entonces, no hay ningún corpus con normas incompatibles porque dichos corpora serían irracionales y vulnerarían el principio kantiano. Replicando a ALCHOURRÓN y BULYGIN (2015, primero publicado en 1989), vON WRIGHT insiste (VON WRIGHT 1989: 876): «El caso en el que la misma autoridad emite prescripciones inconsistentes (sin derogar ninguna de ellas) es una instancia de irracionalidad. Uno no puede "querer" racionalmente cosas contradictorias» ${ }^{10}$.

Pero es más, la irracionalidad de un corpus así se muestra en dos aspectos complementarios: $a$ ) es de imposible cumplimiento para sus destinatarios, para los sujetos normativos, porque les exige realizar estados de cosas imposibles y $b$ ) no es apto para justificar decisiones individuales, porque sea cual sea la decisión individual estaría justificada la norma individual en un corpus inconsistente, y por tanto, no sirve para el fin que le es propio: discriminar entre decisiones justificadas y no justificadas.

ALCHOURRÓN y BULYGIN aceptan este extremo pero creen que la racionalidad no puede ser condición de la existencia ni de las normas ni de los corpora normativos. En Normative Systems (ALCHOURRÓN y BULYGIN 1971: 57) introducen la noción de sistema puramente normativo, un sistema que carece de consecuencias fácticas. Antes de seguir con ello hay que recordar que las normas en dicho libro son concebidas

10 En este texto, creo que, con razón, VON WRIGHT (1989: 877) también muestra cómo los autores argentinos marginan, al aceptar la pluralidad de autoridades y las antinomias, el problema de la unidad o identidad de los sistemas normativos. 
como enunciados que correlacionan casos con soluciones (normas condicionales) y, por lo tanto, dos normas incompatibles (en el sentido de que correlacionan el mismo caso con soluciones incompatibles) implican una proposición fáctica, la negación del antecedente del condicional normativo. Entonces aceptan que (1971: 63) un sistema normativo inconsistente podría ser calificado de irracional y, en este sentido, la consistencia es un ideal racional. Pero añaden: «Conjuntos normativos inconsistentes no son tan raros, como todo jurista sabe por experiencia (...). Pero no parece que haya buenas razones para limitar la referencia del término "sistema" a los conjuntos consistentes, a menos que por "sistema normativo" entendamos un sistema normativo puro, es decir, un sistema que carezca de consecuencias fácticas».

Es claro que ALCHOURRÓn y BuLYGIN llevan razón si pensamos en la posibilidad de que las autoridades, incluso la misma autoridad, se comporten irracionalmente dictando normas incompatibles. Y si la noción de una prescripción depende únicamente de haber sido el resultado de un acto serio de una autoridad, entonces su conclusión se mantiene. Es más, si los corpora solo son conjuntos de normas promulgadas, entonces puede haber corpora inconsistentes. Sin embargo, y aquí reside parte del valor de la contribución de VON WRIGHT, no veo porqué debamos aceptar esta noción de existencia de normas. El autor finlandés nos ha dado buenas razones para aceptar la idea de que las normas existen de modo similar a como existen las promesas, no basta la pronunciación de ciertas palabras para su existencia. Con esta conjetura, podemos argüir que el principio kantiano es una condición de existencia de las normas y, como una de sus consecuencias, que en los corpora normativos no hay normas incompatibles.

Sea como fuere, deseo acabar señalando que esta contribución debe ser leída como un mero comentario al margen del ensayo de ALCHOURRÓN y BULYGIN, escrito en 1973, aunque debió esperar hasta 1989 para ser publicado, un ensayo que, como tantos de los suyos y como muchos de VON WRIGHT, a uno le habría gustado escribir.

\section{REFERENCIAS BIBLIOGRÁFICAS}

Alchourrón, C. E., 1996: «Para una lógica de las razones prima facie», Análisis filosófico, 16: 113-124.

Alchourrón, C. E., y Bulygin, E., 1971: Normative Systems, New York-Wien: Springer.

- 2015: «VON Wright on Deontic Logic and the Philosophy of Law», en E. Bulygin, C. BERnal, C. Huerta, T. Mazzarese, J. J. Moreso, P. Navarro y S. Paulson (eds.), Essays in Legal Philosophy, Oxford: Oxford University Press, cap. 5. Originalmente en P. A. ScHILPP y L. E. HaHn (eds.), The Philosophy of Georg Henrik von Wright, The Library of Living Philosophers, vol. 19, La Salle, Ill.: Open Court, 1989, 665-693; en español en C. E. ALCHOURRÓN; E. Bulygin, Análisis lógico y Derecho, Madrid: Centro de Estudios Constitucionales, 1991, 69-102.

Celano, B., 1994: Dialettica della gisutificazione prattica. Saggio sulla legge di Hume, Torino: Giappichelli.

HERnÁNDEZ MARÍn, R., 1986: Historia de la filosofía del derecho contemporánea, Madrid: Tecnos. HoRWICH, P., 1990: Truth, Oxford: Basil Blackwell.

KanT, I., 1781-1787: Kritik der reinen Vernunft, Akademieausgabe. Gesammelten Werken, III, IV. bttps://korpora.zim.uni-duisburg-essen.de/kant/verzeichnisse-gesamt.btml. 
- (1796): «Verkündigung des nahen Abschlusses eines Tractats zum ewigen Frieden in der Philosophie», Akademieausgabe. Gesammelten Werken, VIII. bttps://korpora.zim.uni-duisburg-essen.de/kant/verzeichnisse-gesamt.html.

McConell, T., 2014: «Moral Dilemmas», en E. N. ZaLTA (ed.), The Stanford Encyclopedia of Philosophy, Fall 2014 Edition, URL = http://plato.stanford.edu/archives/fall2014/entries/ moral-dilemmas/.

Moreso, J. J.; Navarro, P., E., y Redondo, M. C., 2001: «Sobre la lógica de las lagunas en el derecho», Crítica: Revista Hispanoamericana de Filosofía, 33: 47-73.

- 2002: «Legal Gaps and Conclusive Reasons», Theoria: a Swedish journal of philosophy, 68: 51-65.

- 2003: «Bivalencia, antinomias y contradicciones. Un ensayo sobre Truth-Logics de G. H. VON WRIGHT», Doxa, 26: 127-144.

Navarro, P. E., y Rodríguez, J. L., 2014: Deontic Logic and Legal Systems, Cambridge: Cambridge University Press.

STERn, R., 2004: «Does “Ought” Imply “Can”? And Did Kant Think It Does?», Utilitas, 16: 42-61.

Stoljar, D., y Damnjanovic, N., 2014: «The Deflationary Theory of Truth», en E. N. ZaLTA (ed.), The Stanford Encyclopedia of Philosopby (Fall 2014 Edition), URL = http://plato. stanford.edu/archives/fall2014/entries/trutb-deflationary/.

Von Wright, G. H., 1951: «Deontic Logic», Mind, 60: 1-15.

- 1963: Norm and Action. A Logical Enquiry, London: Rouletdge \& Kegan Paul.

- 1983a: «The Foundation of Norms and Normative Propositions», en G. H. vON WrighT (ed.), Practical Reason. Philosophical Papers. Volume I, Oxford: Basil Blackwell, 67-82.

— 1983b: «On Promises», en G. H. vON Wright (ed.), Practical Reason. Philosophical Papers. Volume I, Oxford: Basil Blackwell, 83-99.

- 1983c: «Norms, Truth and Logic», en G. H. vON Wright (ed.), Practical Reason. Philosophical Papers. Volume I, Oxford: Basil Blackwell, 130-209.

- 1984a: «Demystifying Propositions», en G. H. vON Wright (ed.), Truth, Knowledge, and Modality. Philosophical Papers. Volume III, Oxford: Basil Blackwell, 14-25.

- 1984b: «Truth and Logic», en G. H. vON Wright (ed.), Truth, Knowledge, and Modality. Philosophical Papers. Volume III, Oxford: Basil Blackwell, 26-41.

- 1989: «A Reply to My critics», en P. A. SchiLPP y L. E. HaHn (eds.), The Philosophy of Georg Henrik von Wright, The Library of Living Philosophers, vol. 19, La Salle, Ill.: Open Court, 731-887.

Williams, B., 1965: «Ethical Consistency», Proceedings of the Aristotelian Society, Supplement, 39: 103-124.

Williamson, T., 2004: Vagueness, London: Routledge. 